\title{
Evaluating the Interactive Effect of COVID-19 Worry and Loneliness on Mental Health Among Young Adults
}

\author{
Nubia A. Mayorga ${ }^{1} \cdot$ Tanya Smit $^{1} \cdot$ Lorra Garey $^{1} \cdot$ Alexandra K. Gold $^{4} \cdot$ Michael W. Otto $^{4} \cdot$ Michael J. Zvolensky $^{1,2,3} \mathbb{C}$
}

Accepted: 13 July 2021 / Published online: 25 July 2021

(c) The Author(s), under exclusive licence to Springer Science+Business Media, LLC, part of Springer Nature 2021

\begin{abstract}
Background Young adults are overrepresented in terms of adverse mental health problems related to COVID-19. Emerging work has identified worry about the consequences and trajectory of COVID-19 and loneliness as important factors in mental health during the pandemic. However, the main and interactive effects of worry about COVID-19 and loneliness have not been explored in one overarching model in relation to mental health problems among young adults.

Methods The present study therefore evaluated loneliness and COVID-19 related worry in terms of anxiety, stress, and depression among young adults ( 209 college students, $76.1 \%$ female, $M_{\text {age }}=22.99$ years, $S D=5.25$ ) recruited to participate in an online survey study.

Results Results indicated a significant interaction between COVID-19 worry and loneliness for each criterion variable (depression: $b=.01, S E=.003, t=2.86, p=.01$; anxiety: $b=.01, S E=.002, t=2.36, p=.02$; stress: $b=.01, S E=.003, t=2.54$, $p=.01$ ), such that worry was more strongly related to each mental health outcome among those that endorsed higher levels of loneliness.

Conclusion The current findings suggest loneliness is related to negative mental health symptoms among young adults experiencing COVID-19 related worry. The current findings provide initial empirical evidence for the impact of COVID19 worry on mental health among young adults experiencing loneliness. Future research may benefit from exploring how COVID-19 worry and loneliness interplay over time.
\end{abstract}

Keywords COVID-19 · Worry · Loneliness · Young Adults · Pandemic $\cdot$ Mental Health · Depression · Anxiety $\cdot$ Stress

\section{Introduction}

Coronavirus disease 2019 (COVID-19) has had a detrimental physical and mental health impact globally (Resnick et al., 2020; Troyer et al., 2020; Zvolensky et al., 2020). In the United States (U.S.), over 20 million people have been infected with COVID-19 and over 400,000 have died from

Michael J. Zvolensky

mjzvolen@central.uh.edu

1 Department of Psychology, University of Houston, 3695 Cullen Blvd., 126 Heyne Building, Houston, TX 77204, USA

2 Department of Behavioral Science, The University of Texas MD Anderson Cancer Center, Houston, TX, USA

3 HEALTH Institute, University of Houston, Houston, TX, USA

4 Department of Psychology \& Brain Sciences, Boston University, Boston, MA, USA
COVID-19-related medical complications (February 2021; WHO Coronavirus Disease (COVID-19) Dashboard). In addition to COVID-19-related physical health problems, there has been a significant increase in mental health symptoms and disorders associated with the COVID-19 pandemic (Gallagher et al., 2020; Salari et al., 2020), particularly among populations 'at risk' for elevated psychological distress, such as young adults (aged 18-25; Minds, 2020).

Young adults have endorsed some of the highest rates of depression, anxiety, and overall stress during the pandemic (Kujawa et al., 2020). For instance, up to $60 \%$ of young adults have reported symptoms of psychological distress in response to the outbreak of COVID-19 (Martinez \& Nguyen, 2020). Among college students, approximately $45 \%$ have reported "high" levels of psychological impact (i.e., symptoms associated with negative mental health outcomes), $17.4 \%$ of students endorsed increased anxiety, and $14.6 \%$ of students reported greater stress $(N=2534$; Browning et al., 2021). Other national surveys have suggested young adults 
have experienced up to a $55 \%$ increase in stress related to COVID-19 (Palsson \& Ballou, 2020; Stress in America ${ }^{\mathrm{TM}}$, 2020: Stress in the Time of COVID-19, Volume One, 2020). Demographic and behavioral factors associated with the pandemic, such as sex, financial stress, disruption of routine, boredom, and other daily life stressors (e.g., virtual school) have been identified as factors related to observed mental health stress among young adults (Ettman et al., 2020; Panchal et al., 2020). Although these data provide initial evidence for sociodemographic factors related to poor mental health outcomes among this group, non-COVID-19 related research on anxiety and depression among young adults has emphasized the importance of identifying individually-based and targetable mechanisms that amplify the severity of mental health symptoms (Bentley et al., 2018; Ehring \& Behar, 2020; Wolitzky-Taylor et al., 2016).

Individual differences in worry may serve to amplify emotional vulnerability states among young adults during the pandemic. Worry reflects a relatively stable state of apprehension and negative expectations about future events (Borkovec, 1985) and is targetable through intervention (Topper et al., 2017). Worry is a risk factor for depression and related negative emotional symptoms (Muris et al., 2005). Among young adults, higher levels of worry are associated with increased depression (Zvolensky et al., 2019), anxiety (Gana et al., 2001), and stress (Roussis \& Wells, 2008; Wells \& Carter, 2001). Emerging work investigating COVID-19 related worry (i.e., worry that is rooted in the uncertainty that surrounds the trajectory and consequences of COVID-19) has shown that worry about the pandemic is associated with poorer mental health (Kleinberg et al., 2020; Wang et al., 2020) and greater substance use (Rogers et al., 2020). Additionally, worry during the pandemic is related to higher anxiety, anxiety sensitivity, and depression (Baiano et al., 2020), and COVID-19 stress syndrome is conceptualized, in part, on the basis of the tendency to worry about pandemic-related factors (Taylor, 2020). Certain demographic factors (e.g., sex; Van der Vegt \& Kleinberg, 2020) have been shown to influence the degree of experienced worry, but no work has evaluated the relation between individual differences in COVID-19 related worry and other emotional vulnerability factors that contribute to worse psychological outcomes among young adults.

Loneliness represents another emotional vulnerability factor that may be relevant to explore among young adults in the context of anxiety, depression, and stress during the COVID-19 pandemic. Loneliness is characterized as a negative emotional factor that arises when there is perceived discrepancy between current and desired states of an individual's social relationships (David Perlman, 1982). Greater levels of loneliness are associated with greater anxiety (Moeller \& Seehuus, 2019) and depression (Cacioppo et al., 2006; Stessman et al., 2014). During the COVID-19 pandemic, stay-at-home orders and social distancing guidelines may increase the risk of loneliness (Marroquín et al., 2020). Importantly, the experience of loneliness that occurs as a consequence to the COVID-19 pandemic may span beyond the physical separation from others but encompasses a lack of emotionally intimate connection with friends and family. Some work has found that the association between loneliness and depression among young adults is especially strong during the COVID-19 pandemic (Liu et al., 2020). Recent non-COVID-19 research has indicated that the effect of loneliness on negative mental health outcomes may be exacerbated by other vulnerability factors, such as worry (Anyan et al., 2020). The extent to which such synergistic patterns extend to mental health outcomes during the pandemic, however, remain unknown.

Theoretically, individual differences in worry about COVID-19 may be more strongly related to negative mental health symptoms among college students who experience loneliness. Specifically, worry may provide the individual with a false sense of control, as they "prepare" themselves for various aversive future events (Dugas et al., 2004). Worry is differentiated from loneliness that reflects anxious apprenhension about future events whereas loneliness reflects $\mathrm{s}$ a state of emotional discomfort that an individual faces at the realization of desiring social connection and the inability to attain it. In this sense, loneliness may increase the salience for worrying as the individual is depleted of pre-COVID-19 adaptive coping strategies (e.g., social gatherings, seeking advice from peers; Hoffart et al., 2020) and worry may increase the intensity of loneliness as it may amplify the emotional distress they fear will continue in the future.Thus, these factors have the potential to interact with one another to in terms of stress, anxiety, and depression among young adults, such that those who are higher in worry and higher in loneliness may be more likely to experience increased stress, increased anxiety, and increased depression.

The aim of the current study was to evaluate the potential interactive effect of individual differences in worry and loneliness on anxiety, stress, and depression among young adults. It was hypothesized that greater levels of COVID-19 related worry and loneliness would be related to higher levels of anxiety, stress, and depression symptoms.

\section{Method}

\section{Participants}

The present study included 209 college students $(76.1 \%$ female, $M_{\text {age }}=22.99$ years, $S D=5.25$, range $=18-31$ ) recruited to participate in an online survey study for course credit. Participants had to be currently enrolled in a psychology course, had to be at least 18 years old, and had to be able 
to provide informed consent. Exclusion criteria included being a non-English speaker (to ensure comprehension of the study questions).

The present sample consisted of White/Caucasian (40.7\%) individuals, followed by $25.4 \%$ Asian, $12.4 \%$ Black/ African American, 8.1\% 'Other', 7.2\% 'more than one race,' $0.5 \%$ Alaska Native or Native American, and $0.5 \%$ Native Hawaiian or Pacific Islander; 5.3\% of the sample declined to provide their race. Regarding ethnicity, $37.8 \%$ of participants identified as being Spanish/Hispanic/Latinx. Eight participants (4.8\%) reported ever being diagnosed with COVID-19. Regarding education, $8.4 \%$ were freshman (1st year), $16.4 \%$ were sophomore (2nd year), 31.4\% were junior (3rd year), $35.8 \%$ were senior ( 4 th year), $5.3 \%$ were 5 th year or higher, and $2.7 \%$ declined to answer. Regarding employment, $54.5 \%$ of the sample reported being currently employed.

\section{Measures}

\section{Demographics Questionnaire}

Participants provided demographic information such as their gender, age, race, ethnicity, and education. Age (Lorem et al., 2017), employment-related stress (McDonough, 2000), gender (Abrams et al., 2019), race, and ethnicity (Spanish/Hispanic/Latinx or non-Spanish/HispanicLatinx; General, 2001; Hooper et al., 2020) were all included as covariates within the model.

\section{Employment-Related Stress}

A single item created by the current research team (i.e., How stressed are you about your current employment situation?") aimed to assess the stress related to an individual's employment status. Participants rated this item on a scale from 1 (Not at all) to 10 (Extremely). Employment-related stress was included in all models as a covariate.

\section{UCLA Loneliness Scale (Version 3)}

The UCLA Loneliness Scale (Russell, 1996) is a 20-item self-report measure assessing loneliness, conceptualized as an unpleasant subjective internal experience during which an individual perceives their social network as being insufficient (Daniel Perlman \& Peplau, 1982). Participants are asked to rate how often they relate to a series of items pertaining to loneliness (e.g., "How often do you feel that you lack companionship?") on a 1 (Never) to 4 (Always) scale. The scale yields a total score, and higher scores indicate higher levels of loneliness. Past work has demonstrated that the UCLA Loneliness Scale has sound psychometric properties (Hartshorne, 1993). In the current sample, the loneliness total score was used (Cronbach's $\alpha=0.72$ ).

\section{COVID-19 Worry Index}

The COVID-19 Worry Index is a 15 -item measure created by the current research team (Zvolensky et al., 2020) and is based off of established measures of worry (Meyer et al., 1990). This measures assesses worry specific to the COVID19 pandemic across various topics, including but not limited to, health (e.g., "I worry that I am going to become seriously ill due to COVID-19"), social relationships ("I am worried I will lose friends due to social distancing"), and finances ("I am worried I will not be able to financially provide for myself or my family during this time"; Wilson et al., 2020). For each item, participants were asked to rate how worried they feel about each item on a scale from 1 (Not at all) to 10 (A great deal). A total COVID-19 worry score was used in the current study as the predictor in all models and this subscale demonstrated excellent internal consistency in the current sample (Cronbach's $\alpha=0.93$ ).

\section{Depression Anxiety Stress Scales (DASS-21)}

The DASS-21(Antony et al., 1998) is 21-item self-report measure assessing symptoms of anxiety and depression. Participants are asked to rate several statements on a scale from 0 (Did not apply to me at all) to 3 (Applied to me very much, or most of the time). This measure yields three scales: the depression scale, which measures dysphoric mood (e.g., "I felt that life was meaningless"), the anxiety scale, which measures symptoms of physical arousal, panic attacks, and fear (e.g., "I felt scared without any good reason"), and the stress scale, which measures symptoms related to tension, irritability, and overreacting to stressful events (e.g., "I was intolerant of anything that kept me from getting on with what I was doing"). The three DASS-21 scales were utilized as the criterion variables in our models and demonstrated good to excellent internal consistency (depression: Cronbach's $\alpha=0.93$; anxiety: Cronbach's $\alpha=0.88$; stress: Cronbach's $\alpha=0.88$ ).

\section{Data Analysis}

Analyses were conducted using SPSS version 25. First, sample descriptive statistics and zero-order correlations among study variables were examined. Second, to evaluate main and interactive effects of COVID-19 worry and loneliness, three separate hierarchical regression analyses were conducted for anxiety, depression, and stress as criterion variables. Covariates entered in the first step of each model included age, employment-related stress, gender, race, and ethnicity (Spanish/Hispanic/Latinx or non-Spanish/HispanicLatinx). Race and ethnicity were dichotomized as follows: race $(0=$ White, $1=$ Non-White $)$ and ethnicity $(0=$ Not Spanish/Hispanic/Latinx, $1=$ Spanish/Hispanic/Latinx). In 
Table 1 Descriptive statistics and correlations among variables

\begin{tabular}{|c|c|c|c|c|c|c|c|c|c|c|c|}
\hline & Mean $/ n$ & $S D / \%$ & 1 & 2 & 3 & 4 & 5 & 6 & 7 & 8 & 9 \\
\hline 1. Depression & 11.30 & 11.17 & - & & & & & & & & \\
\hline 2. Anxiety & 8.78 & 9.54 & $0.67^{* * *}$ & - & & & & & & & \\
\hline 3. Stress & 13.12 & 10.29 & $0.78^{* * * *}$ & $0.74^{* * * *}$ & - & & & & & & \\
\hline 4. COVID-19 worry & 47.89 & 19.55 & $0.26^{* * *}$ & $0.35^{* * *}$ & $0.32^{* * *}$ & - & & & & & \\
\hline 5. Loneliness (UCLA) & 45.75 & 13.05 & $0.57^{* * *}$ & $0.37^{* * * *}$ & $0.48^{* * * *}$ & $0.22^{* *}$ & - & & & & \\
\hline 6. Age & 23.17 & 5.59 & $-0.16^{*}$ & -0.09 & -0.09 & -0.03 & -0.11 & - & & & \\
\hline 7. Employment-related stress & 2.59 & 1.29 & $0.23^{* *}$ & $0.26^{* * * *}$ & $0.21^{* *}$ & $0.48^{* * *}$ & $0.19^{* *}$ & 0.10 & - & & \\
\hline 8. Gender (\% Female) & $125(n)$ & $75.8 \%$ & 0.07 & 0.06 & $0.15^{*}$ & $0.21^{* *}$ & 0.10 & 0.10 & $0.19^{* *}$ & - & \\
\hline 9. Race (\% Non-White) & $127(n)$ & $56.2 \%$ & -0.04 & -0.07 & 0.05 & -0.06 & -0.05 & 0.13 & 0.04 & 0.10 & - \\
\hline 10. Ethnicity (\% Hispanic) & $64(n)$ & $38.8 \%$ & -0.03 & 0.02 & -0.02 & -0.002 & -0.01 & -0.07 & -0.07 & -0.13 & $-0.53^{* * *}$ \\
\hline
\end{tabular}

$N=165 ;{ }^{* * *} p<.001,{ }^{* *} p<.01,{ }^{*} p<.05$. Depression, Anxiety, and Stress: Depression, Anxiety, and Stress Scales - 21 (DASS-21; Antony et al., 1998); COVID-19 worry: COVID-19 Worry Index; Loneliness: UCLA Loneliness Scale (Russell, 1996); Employment-related stress: "How stressed are you about your current employment situation?" 1 (Not at all) to 10 (Extremely); Gender: $0=$ Male, $1=$ Female; Race: $0=$ White, 1 = Non-White; Ethnicity: 0= Not Spanish/Hispanic/Latinx, $1=$ Spanish/Hispanic/Latinx

step 2, COVID-19 worry and loneliness were simultaneously entered. In step 3, the interaction of COVID-19 worry and loneliness was added. Planned simple slope post hoc analyses were conducted using high and low values (e.g. \pm 1 SD from the mean) of the moderator variable to examine the association of COVID-19 worry and depression, anxiety, and stress as a function of loneliness. In addition, the Johnson-Neyman (JN) technique (Aiken et al., 1991) was used to statistically identify regions of values in COVID-19 worry where the effect of loneliness was statistically significant, per recommendations of Hayes (2013). Simple slopes and the JN procedure were conducted using the PROCESS macro for SPSS (Hayes, 2012). Effect sizes for interactions were evaluated using unique variance explained $\left(\Delta R^{2}\right)$. Lastly, to supplement our results, analyses were re-run using unadjusted models. ${ }^{1}$

\section{Results}

\section{Descriptive Statistics}

Descriptive statistics and bivariate correlations are presented in Table 1. COVID-19 worry was significantly and positively related to depression $(r=0.29, p<0.001)$, anxiety $(r=0.35, p<0.001)$, and stress $(r=0.32, p<0.001)$. Loneliness was significantly and positively related to depression ( $r=0.57, p<0.001)$, anxiety $(r=0.37, p<0.001)$, and stress $(r=0.48, p<0.001)$. COVID-19 worry and loneliness were

\footnotetext{
${ }^{1}$ Results were consistent with the original pattern of findings in that for all criterion variables, COVID-19 worry and loneliness contributed to statistical significant overall model fit and the interaction of COVID-19 and loneliness explained a significant amount of variance in for the criterion variables.
}

significantly and positively related $(r=0.22,4 \%$ shared variance with one another, $p=0.02)$. The criterion variables were interrelated (range of $r$ 's $0.67-0.78, p<0.001$ ).

\section{Primary Analyses}

For depression, covariates entered in the first step accounted for a significant (statistically significant) amount of variance on the criterion variable $(F[5,203]=4.09, p=0.001$, $R^{2}=0.09$; see Table 2). Employment stress $(p<0.001)$ and age $(p=0.01)$ each had a significant effect. Step two accounted for significantly more variance in depression $\left(\Delta R^{2}=0.28, p<0.001\right)$ and a significant main effect emerged for COVID-19 worry. Step three accounted for significantly more variance in depression $\left(\Delta R^{2}=0.03, p=0.01\right)$. There was a significant interaction of COVID-19 worry and loneliness $(p=0.01)$. Simple slope analysis revealed COVID-19 worry related to depressive symptoms among those at higher levels of loneliness $(b=0.17, S E=0.05, p<0.001)$; COVID19 worry was not related to depressive symptoms among those at lower levels of loneliness $(b=-0.01, S E=0.05$, $p=0.80$; see Fig. 1).

For anxiety, covariates entered in the first step accounted for a significant amount of variance $(F[5,203]=3.46$, $p=0.01, R^{2}=0.08$; see Table 2). Employment stress $(p<0.001)$ was a significant predictor. Step two accounted for significantly amount more variance in anxiety $\left(\Delta R^{2}=0.14, p<0.001\right)$ and a significant main effect emerged for COVID-19 worry. Step three accounted for significantly more variance in anxiety $\left(\Delta R^{2}=0.02, p=0.01\right)$. There was a significant interaction of COVID-19 worries and loneliness $(p=0.02)$. Simple slope analysis revealed that COVID-19 worry related to anxiety symptoms among those at higher levels of loneliness $(b=0.19, S E=0.05, p<0.001)$; COVID19 worry was not related to anxiety symptoms among those 
Table 2 Regression model

Depression

\begin{tabular}{|c|c|c|c|c|c|c|}
\hline & $B$ & $S E$ & $t$ & $p$ & $s r^{2}$ & $R^{2}$ change \\
\hline Step 1 & & & & & & 0.09 \\
\hline Employment stress & 2.02 & 0.57 & 3.52 & $<0.001$ & 0.06 & \\
\hline Age & -0.38 & 0.14 & -2.75 & 0.01 & 0.03 & \\
\hline Gender & 1.31 & 1.73 & 0.78 & 0.45 & 0.003 & \\
\hline Race & -0.12 & 0.47 & -0.25 & 0.81 & $<0.001$ & \\
\hline Ethnicity & 0.66 & 1.72 & 0.38 & 0.70 & 0.001 & \\
\hline Step 2 & & & & & & 0.28 \\
\hline COVID-19 worry & 0.08 & 0.04 & 2.12 & 0.04 & 0.01 & \\
\hline Loneliness & 0.44 & 0.05 & 8.91 & $<0.001$ & 0.25 & \\
\hline Step 3 & & & & & & 0.03 \\
\hline COVID-19 worry*Loneliness & 0.01 & 0.003 & 2.86 & 0.01 & 0.02 & \\
\hline \multicolumn{7}{|l|}{ Anxiety } \\
\hline & $b$ & $S E$ & $t$ & $p$ & $s r^{2}$ & $R^{2}$ change \\
\hline Step 1 & & & & & & 0.08 \\
\hline Employment stress & 1.79 & 0.49 & 3.67 & $<0.001$ & 0.06 & \\
\hline Age & -0.19 & 0.19 & -1.59 & 0.11 & 0.01 & \\
\hline Gender & 0.74 & 1.47 & 0.50 & 0.62 & 0.001 & \\
\hline Race & -0.32 & 0.40 & -0.81 & 0.42 & 0.003 & \\
\hline Ethnicity & 0.05 & 1.46 & 0.04 & 0.97 & $<0.001$ & \\
\hline Step 2 & & & & & & 0.14 \\
\hline COVID-19 worry & 0.11 & 0.03 & 3.40 & 0.001 & 0.04 & \\
\hline Loneliness & 0.21 & 0.05 & 4.51 & $<0.001$ & 0.08 & \\
\hline Step 3 & & & & & & 0.02 \\
\hline COVID-19 worry*Loneliness & 0.01 & 0.002 & 2.37 & 0.02 & 0.15 & \\
\hline \multicolumn{7}{|l|}{ Stress } \\
\hline & $b$ & $S E$ & $t$ & $p$ & $s r^{2}$ & $R^{2}$ change \\
\hline Step 1 & & & & & & 0.07 \\
\hline Employment stress & 1.53 & 0.54 & 2.84 & 0.01 & 0.19 & \\
\hline Age & -0.25 & 0.13 & -1.87 & 0.06 & 0.017 & \\
\hline Gender & 2.86 & 1.63 & 1.75 & 0.08 & 0.12 & \\
\hline Race & 0.33 & 0.44 & 0.75 & 0.45 & 0.05 & \\
\hline Ethnicity & 0.63 & 1.62 & 0.39 & 0.70 & 0.03 & \\
\hline Step 2 & & & & & & 0.22 \\
\hline COVID-19 worry & 0.11 & 0.04 & 3.00 & 0.003 & 0.03 & \\
\hline Loneliness & 0.33 & 0.05 & 6.76 & $<0.001$ & 0.16 & \\
\hline Step 3 & & & & & & 0.02 \\
\hline COVID-19 worry*Loneliness & 0.01 & 0.003 & 2.54 & 0.01 & 0.02 & \\
\hline
\end{tabular}

$N=319$; New variables are represented in each step, however, all previous variables were retained

at lower levels of loneliness $(b=0.04, S E=0.04, p=0.32$; see Fig. 2).

For stress, covariates entered in the first step accounted for a significant amount of variance $(F[5,1203]=3.19, p=0.01$, $R^{2}=0.07$; see Table 2). Employment stress $(p=0.01)$ was the only significant predictor. Step two accounted for significantly more variance in stress $\left(\Delta R^{2}=0.22, p<0.001\right)$, and COVID-19 worry emerged as a significant predictor.
Step three accounted for significantly more variance in stress $\left(\Delta R^{2}=0.02, p=0.01\right)$. There was a significant interaction of COVID-19 worries and loneliness $(p=0.01)$. Simple slope analysis revealed that COVID-19 worry related to stress symptoms among those at higher levels of loneliness ( $b=0.19, S E=0.05, p<0.001)$; COVID-19 worry was not related to stress symptoms among those at lower levels of loneliness ( $b=0.03, S E=0.05, p=0.55$; see Fig. 3). 
Fig. 1 Interaction of COVID19 worry and loneliness on depression
Fig. 2 Interaction of COVID-19 worry and loneliness on anxiety
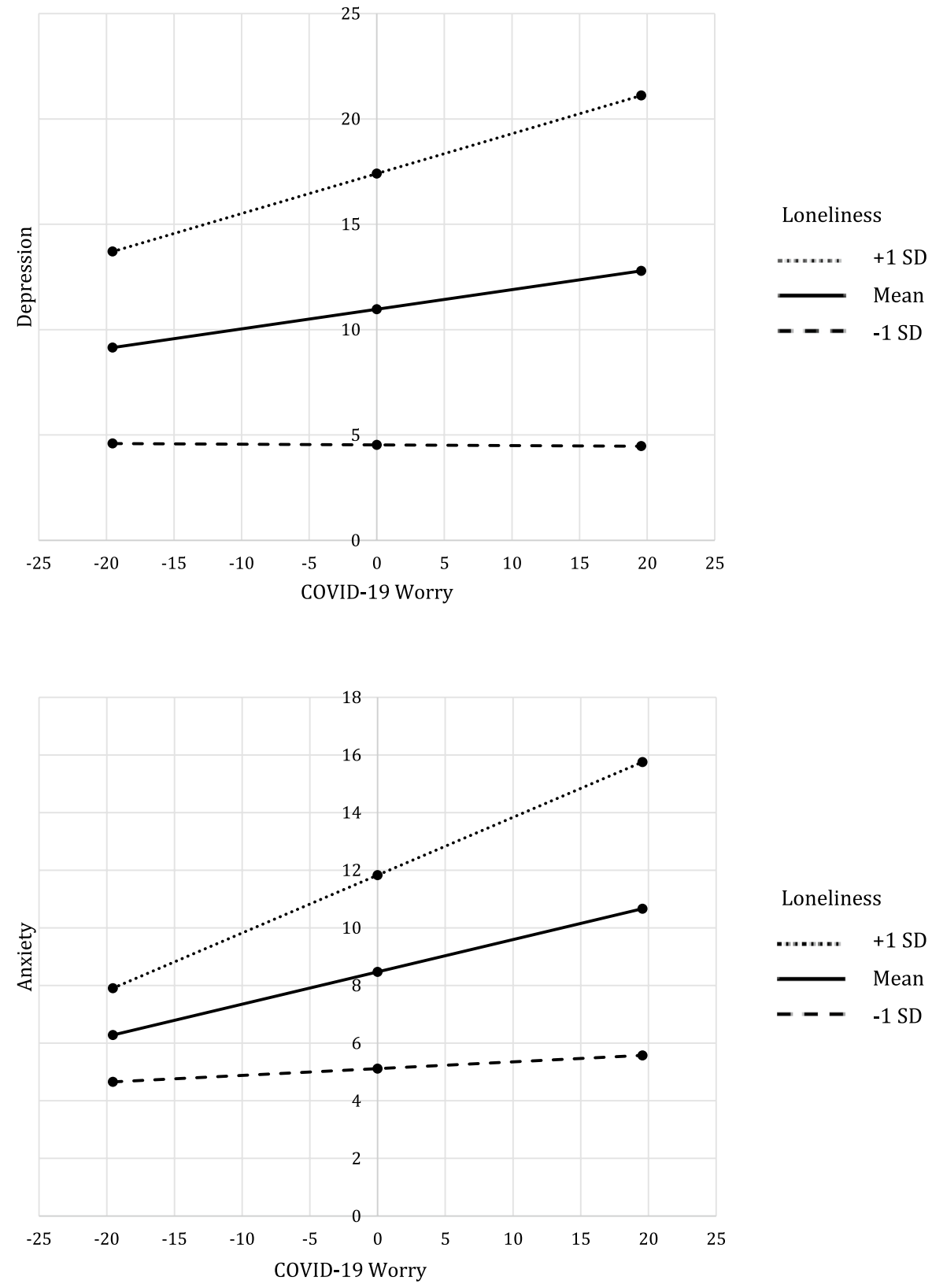

\section{Discussion}

This study aimed to investigate the interactive effect of individual differences in COVID-19 related worry and loneliness in relation to depression, anxiety, and stress among young adults. Findings were consistent with our initial hypotheses. Specifically, there was a significant interactive effect for COVID-19 worry and loneliness for each criterion variable, accounting for an additional 2-3\% of the variance explained over the theoretically relevant covariates of age, employment-related stress, gender, race, and ethnicity. Inspection of the forms of the significant interactions indicated that individuals reporting co-occurring high levels of COVID-19 worry and loneliness evidenced the greatest levels of emotional distress. These results highlight the synergistic role of COVID-19 worry and loneliness beyond the respective main effects for the studied mental health variables. The size of the observed significant interaction effects was small, according to conventional standards (Cohen et al., 2013). However, given the magnitude of variance accounted for at steps 1 and 2 (ranging from $22 \%$ to $37 \%$ of variance), it is noteworthy that the interactive effects enhanced the model's predictive power (Abelson, 1985). Thus, the overall pattern of findings were consistent with theoretical models suggesting that there may indeed be clinically relevant interplay between COVID-19 worry and loneliness in regard to negative emotional symptoms among young adults. 
Fig. 3 Interaction of COVID-19 worry and loneliness on stress

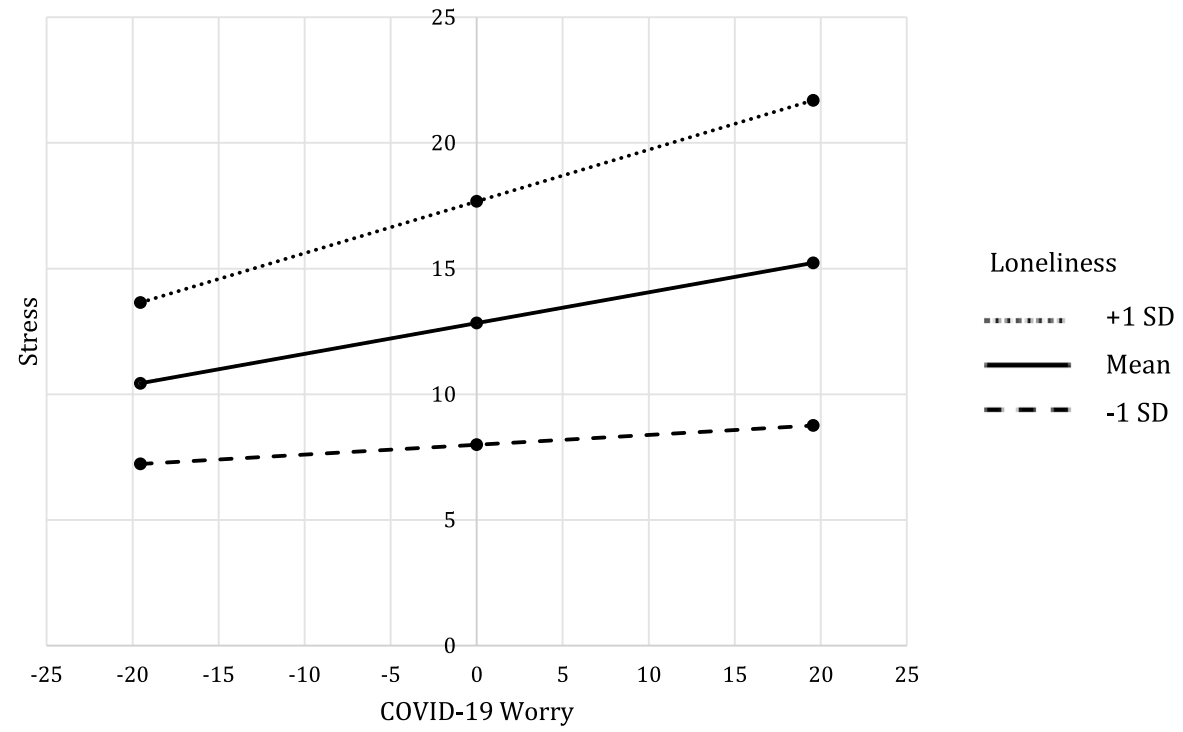

It is important to note that these data form part of a preliminary model and suggest COVID-19 worry on mental health during the pandemic is likely to be exacerbated in the presence of increased loneliness among young adults. As the ongoing pandemic and associated regulations that limit social interactions are likely to continue for some time ( "Study Sees Need for Some Social Distancing into 2022 to Curb Coronavirus," 2020), worry and loneliness may remain elevated in the general population. Therefore, ongoing work may benefit from continued investigation of worry and loneliness as factors impacting mental health outcomes both during and after the pandemic.

In terms of main effects, COVID-19 related worry emerged as an independent predictor and related to symptoms of increased stress, anxiety, and depression. These data suggest that among young adults, worry within the context of the ongoing pandemic may exacerbate risk for negative mental health outcomes. This association has been evident in the general population (Rogers et al., 2020), but had yet to be investigated specifically among young adults. Of note, even though loneliness was related to worry in terms of the criterion symptoms, loneliness alone did not reach statistical significance as a main effect predictor when evaluated in the context of worry. This pattern of associations supports the conceptualization of loneliness as both sharing variance with and also moderating the more primary effects of worry. The current sample reported a level of loneliness that would be elevated compared to normative data (Morhan-Martin and Schumacher, 2003), underscoring the prevalence of loneliness during the pandemic and suggesting that greater attention could be focused on this construct. In terms of COVID-19 worry, the current results extend past work by highlighting that
COVID-19 worry is related to negative mental health outcomes among young adults (Rogers et al., 2020).

These findings have important clinical implications. Results suggest worry about the outcomes and consequences of the current pandemic may be a risk factor for anxiety, stress, and depression, particularly in the context of loneliness. Thus, interventions could seek to address COVID-19 related worry and loneliness in one overarching therapeutic model. For example, there may be value in providing psychoeducation for young adults on the interactive nature of worry related to the pandemic and the loneliness that may exacerbate such worry. Along with psychoeducation, clinicians may seek to provide young adults with strategies to lower levels of worry (e.g., structuring worry time, cognitive restructuring) and promote activities that combat loneliness (e.g., outdoor activities, virtual or socially distant interactions with friends, peer support and disclosure; Steger \& Kashdan, 2009). However, it is important to consider elevated levels of loneliness and worry may encompass a relatively "normal" response to a pandemic and the restrictions that accompany the pandemic. Indeed, elevated levels of worry may serve a protective function in terms of prompting individuals to adhere to guidelines and updated restrictions. Young adults may likely benefit from a nuanced and balanced perspective of theseemotional states, in which worry and loneliness are normalized as responses, while monitoring the threshold for "elevated" worry and loneliness.

The present study has limitations that warrant comment and can serve to better inform future work in this area. First, the study utilized cross sectional data. This type of data collection impedes interpretation of temporal associations across studied variables. Future prospective research is therefore needed to provide better insight into the 
longitudinal nature of the interplay between the COVID-19 worry and loneliness. Second, data was collected based on self-report. Future work may seek to employ multimethod methodology to decrease shared method variance. Third, COVID-19 related worry was evaluated as a unidimensional construct. Future work may benefit from investigating whether certain "areas" of worry are more pronounced among young adults (e.g., finances, health). These investigations may provide a more nuanced understanding of the risk level for negative mental health symptoms associated with specific worries. Finally, no information was gathered on the regulations and social practices of the sample. Evaluating to what degree social distancing restrictions are enforced, whether individuals engage in virtual gatherings, and the level of compliance with distancing guidelines may provide a better understanding on the levels of worry and loneliness among young adults.

Overall, the current findings provide initial empirical evidence for the impact of COVID-19 worry on mental health among young adults experiencing loneliness. Future research may benefit from exploring how COVID-19 worry and loneliness interplay over time.

Funding Research reported in this publication was supported by the National Institute on Minority Health and Health Disparities (NIMHD) of the National Institutes of Health (NIH) to the University of Houston under Award Number U54MD015946. The content is solely the responsibility of the authors and does not necessarily represent the official views of the National Institutes of Health.

\section{Declarations}

Conflict of interest Nubia A. Mayorga, Tanya Smit, Lorra Garey, Alexandra K. Gold, Michael W. Otto, Michael J. Zvolensky declare that they have no conflict of interest is evident for the content of this manuscript, the authors would like to acknowledge the following relationship. Dr. Otto receives compensation as a consultant for Big Health.

Animal Rights No animal studies were carried out by the authors for this article.

Informed Consent All participants provided informed consent prior to participating in this research study.

\section{References}

Abelson, R. P. (1985). A variance explanation paradox: When a little is a lot. Psychological Bulletin, 97(1), 129.

Abrams, L. R., Kalousova, L., \& Fleischer, N. L. (2019). Gender differences in relationships between sociodemographic factors and e-cigarette use with smoking cessation: 2014-15 current population survey tobacco use supplement. Journal of Public Health. https://doi.org/10.1093/pubmed/fdz017

Antony, M. M., Bieling, P. J., Cox, B. J., Enns, M. W., \& Swinson, R. P. (1998). Psychometric properties of the 42-item and 21-item versions of the Depression Anxiety Stress Scales in clinical groups and a community sample. Psychological Assessment, 10(2), 176.

Baiano, C., Zappullo, I., \& Conson, M. (2020). Tendency to worry and fear of mental health during Italy's COVID-19 lockdown. International Journal of Environmental Research and Public Health, 17(16), 5928.

Bentley, K. H., Boettcher, H., Bullis, J. R., Carl, J. R., Conklin, L. R., Sauer-Zavala, S., Pierre-Louis, C., Farchione, T. J., \& Barlow, D. H. (2018). Development of a single-session, transdiagnostic preventive intervention for young adults at risk for emotional disorders. Behavior Modification, 42(5), 781-805.

Borkovec, T. D. (1985). Worry: A potentially valuable concept. Behaviour Research and Therapy, 23(4), 481-482.

Browning, M. H., Larson, L. R., Sharaievska, I., Rigolon, A., McAnirlin, O., Mullenbach, L., Cloutier, S., Vu, T. M., Thomsen, J., \& Reigner, N. (2021). Psychological impacts from COVID-19 among university students: Risk factors across seven states in the United States. PloS One, 16(1), e0245327.

Cacioppo, J. T., Hughes, M. E., Waite, L. J., Hawkley, L. C., \& Thisted, R. A. (2006). Loneliness as a specific risk factor for depressive symptoms: Cross-sectional and longitudinal analyses. Psychology and Aging, 21(1), 140-151. https://doi.org/10.1037/0882-7974. 21.1.140

Cohen, J., Cohen, P., West, S. G., \& Aiken, L. S. (2013). Applied multiple regression/correlation analysis for the behavioral sciences. Routledge.

Dugas, M. J., Schwartz, A., \& Francis, K. (2004). Brief report: Intolerance of uncertainty, worry, and depression. Cognitive Therapy and Research, 28(6), 835-842.

Ehring, T., \& Behar, E. (2020). Transdiagnostic view on worrying and other negative mental content. Generalized Anxiety Disorder and Worrying: A Comprehensive Handbook for Clinicians and Researchers, 43-68.

Ettman, C. K., Abdalla, S. M., Cohen, G. H., Sampson, L., Vivier, P. M., \& Galea, S. (2020). Prevalence of depression symptoms in US adults before and during the COVID-19 pandemic. JAMA Network Open, 3(9), e2019686-e2019686.

Gallagher, M. W., Zvolensky, M. J., Long, L. J., Rogers, A. H., \& Garey, L. (2020). The impact of covid-19 experiences and associated stress on anxiety, depression, and functional impairment in American adults. Cognitive Therapy and Research, 44(6), 1043-1051.

Gana, K., Martin, B., \& Canouet, M.-D. (2001). Worry and anxiety: Is there a causal relationship? Psychopathology, 34(5), 221-229.

General, U. S. (2001). Mental health: Culture, race, and ethnicity. A supplement to mental health: A report of the surgeon general. US Department of Health and Human Services.

Hartshorne, T. S. (1993). Psychometric properties and confirmatory factor analysis of the UCLA Loneliness Scale. Journal of Personality Assessment, 61(1), 182-195.

Hayes, A. F. (2012). PROCESS: A versatile computational tool for observed variable mediation, moderation, and conditional process modeling.

Hayes, N. (Ed.). (2013). Doing qualitative analysis in psychology. Psychology Press.

Hoffart, A., Johnson, S. U., \& Ebrahimi, O. V. (2020). Loneliness and social distancing during the COVID-19 pandemic: Risk factors and associations with psychopathology. Frontiers in Psychiatry, 11, 1297.

Hooper, M. W., Nápoles, A. M., \& Pérez-Stable, E. J. (2020). COVID-19 and racial/ethnic disparities. Jama.

Kleinberg, B., van der Vegt, I., \& Mozes, M. (2020). Measuring emotions in the covid-19 real world worry dataset.

Kujawa, A., Green, H., Compas, B. E., Dickey, L., \& Pegg, S. (2020). Exposure to COVID-19 pandemic stress: Associations with 
depression and anxiety in emerging adults in the United States. Depression and Anxiety, 37(12), 1280-1288.

Liu, C. H., Zhang, E., Wong, G. T. F., \& Hyun, S. (2020). Factors associated with depression, anxiety, and PTSD symptomatology during the COVID-19 pandemic: Clinical implications for US young adult mental health. Psychiatry Research, 290, 113172.

Lorem, G. F., Schirmer, H., Wang, C. E. A., \& Emaus, N. (2017). Ageing and mental health: Changes in self-reported health due to physical illness and mental health status with consecutive cross-sectional analyses. British Medical Journal Open, 7(1), e013629. https://doi.org/10.1136/bmjopen-2016-013629

Marroquín, B., Vine, V., \& Morgan, R. (2020). Mental health during the COVID-19 pandemic: Effects of stay-at-home policies, social distancing behavior, and social resources. Psychiatry Research, 293, 113419.

Martinez, A., \& Nguyen, S. (2020). The Impact of Covid-19 on College Student Well-Being.

McDonough, P. (2000). Job insecurity and health. International Journal of Health Services, 30(3), 453-476. https://doi.org/10.2190/ BPFG-X3ME-LHTA-6RPV

Minds, A. (2020). COVID-19 impact on college student mental health. Activeminds. Org.

Moeller, R. W., \& Seehuus, M. (2019). Loneliness as a mediator for college students' social skills and experiences of depression and anxiety. Journal of Adolescence, 73, 1-13.

Muris, P., Roelofs, J., Rassin, E., Franken, I., \& Mayer, B. (2005). Mediating effects of rumination and worry on the links between neuroticism, anxiety, and depression. Personality and Individual Differences, 39(6), 1105-1111.

Palsson, O. S., \& Ballou, S. (n.d.). The U.S. National Pandemic Emotional Impact Report. 37.

Panchal, N., Kamal, R., Orgera, K., Cox, C., Garfield, R., Hamel, L., \& Chidambaram, P. (2020). The implications of COVID-19 for mental health and substance use. Kaiser Family Foundation.

Perlman, Daniel, \& Peplau, L. A. (1982). Theoretical approaches to loneliness. Loneliness: A Sourcebook of Current Theory, Research and Therapy, 123-134.

Perlman, D. (1982). Loneliness: A sourcebook of current theory, research, and therapy (Vol. 36). Wiley.

Resnick, A., Galea, S., \& Sivashanker, K. (2020). Covid-19: The painful price of ignoring health inequities. BMJ Opinion, 18.

Rogers, A. H., Shepherd, J. M., Garey, L., \& Zvolensky, M. J. (2020). Psychological factors associated with substance use initiation during the COVID-19 pandemic. Psychiatry Research, 293, 113407.

Roussis, P., \& Wells, A. (2008). Psychological factors predicting stress symptoms: Metacognition, thought control, and varieties of worry. Anxiety, Stress, \& Coping, 21(3), 213-225.

Russell, D. W. (1996). UCLA loneliness scale (Version 3): Reliability, validity, and factor structure. Journal of Personality Assessment, 66(1), 20-40.

Salari, N., Hosseinian-Far, A., Jalali, R., Vaisi-Raygani, A., Rasoulpoor, S., Mohammadi, M., Rasoulpoor, S., \& Khaledi-Paveh, B. (2020). Prevalence of stress, anxiety, depression among the general population during the COVID-19 pandemic: A systematic review and meta-analysis. Globalization and Health, 16(1), 1-11.

Steger, M. F., \& Kashdan, T. B. (2009). Depression and everyday social activity, belonging, and well-being. Journal of Counseling Psychology, 56(2), 289.

Stessman, J., Rottenberg, Y., Shimshilashvili, I., Ein-Mor, E., \& Jacobs, J. M. (2014). Loneliness, health, and longevity. Journals of Gerontology Series A: Biomedical Sciences and Medical Sciences, 69(6), 744-750.
Stress in America ${ }^{T M}$ 2020: Stress in the Time of COVID-19, Volume One. (n.d.). Retrieved November 11, 2020, from https://www.apa. org/news/press/releases/stress/2020/report

Study sees need for some social distancing into 2022 to curb coronavirus. (2020, April 14). STAT. https://www.statnews.com/2020/ 04/14/some-social-distancing-may-be-needed-into-2022-to-keepcoronavirus-in-check-new-study-says/

Taylor, S. (2020). COVID stress syndrome: Clinical and nosological considerations. Current Psychiatry Reports, 23(4), 1-7.

Topper, M., Emmelkamp, P. M., Watkins, E., \& Ehring, T. (2017). Prevention of anxiety disorders and depression by targeting excessive worry and rumination in adolescents and young adults: A randomized controlled trial. Behaviour Research and Therapy, 90, 123-136.

Troyer, E. A., Kohn, J. N., \& Hong, S. (2020). Are we facing a crashing wave of neuropsychiatric sequelae of COVID-19? Neuropsychiatric symptoms and potential immunologic mechanisms. Brain, Behavior, and Immunity.

Van der Vegt, I., \& Kleinberg, B. (2020). Women worry about family, men about the economy: Gender differences in emotional responses to COVID-19. International Conference on Social Informatics, 397-409.

Wang, C., Pan, R., Wan, X., Tan, Y., Xu, L., Ho, C. S., \& Ho, R. C. (2020). Immediate psychological responses and associated factors during the initial stage of the 2019 coronavirus disease (COVID-19) epidemic among the general population in China. International Journal of Environmental Research and Public Health, 17(5), 1729.

Wells, A., \& Carter, K. (2001). Further tests of a cognitive model of generalized anxiety disorder: Metacognitions and worry in GAD, panic disorder, social phobia, depression, and nonpatients. Behavior Therapy, 32(1), 85-102. https://doi.org/10.1016/S00057894(01)80045-9

WHO Coronavirus Disease (COVID-19) Dashboard. (n.d.). Retrieved November 1, 2020, from https://covid19. who.int

Wilson, J. M., Lee, J., Fitzgerald, H. N., Oosterhoff, B., Sevi, B., \& Shook, N. J. (2020). Job insecurity and financial concern during the COVID-19 pandemic are associated with worse mental health. Journal of Occupational and Environmental Medicine, 62(9), 686-691. https://doi.org/10.1097/JOM.0000000000001962

Wolitzky-Taylor, K., McBeth, J., Guillot, C. R., Stone, M. D., Kirkpatrick, M. G., Zvolensky, M. J., Buckner, J. D., \& Leventhal, A. M. (2016). Transdiagnostic processes linking anxiety symptoms and substance use problems among adolescents. Journal of Addictive Diseases, 35(4), 266-277.

Zvolensky, M. J., Garey, L., Rogers, A. H., Schmidt, N. B., Vujanovic, A. A., Storch, E. A., Buckner, J. D., Paulus, D. J., Alfano, C., \& Smits, J. A. (2020). Psychological, addictive, and health behavior implications of the COVID-19 pandemic. Behaviour Research and Therapy, 134, 103715.

Zvolensky, M. J., Kauffman, B. Y., Bogiaizian, D., Viana, A. G., Bakhshaie, J., \& Peraza, N. (2019). Worry among Latinx college students: relations to anxious arousal, social anxiety, general depression, and insomnia. Journal of American College Health, 1-8.

Publisher's Note Springer Nature remains neutral with regard to jurisdictional claims in published maps and institutional affiliations. 